\title{
A NOTE ON $G$-INVARIANT FORMS
}

\author{
STEPHEN M. GAGOLA, JR.
}

(Communicated by Ronald Solomon)

\begin{abstract}
If $G$ is a finite group, the reduction $\bmod p$ of a module supporting a nondegenerate $G$-invariant form need not itself support such a form. However, under a suitable hypothesis on the splitting field (quadratric closure) and a carefully chosen lattice within the module (for reduction $\bmod p$ ), this will always be the case. The argument given is elementary and self-contained.
\end{abstract}

Let $K \supseteq R \rightarrow F$ be a " $p$-modular system" for the finite group $G$. That is, $R$ is a valuation domain with maximal ideal $P$, field of fractions $K$, and residue class field $F$ where $K$ and $F$ are splitting fields for $G$ in characteristics 0 and $p$ respectively. Thus, every (finitely generated) $K G$-module $V$ contains an $R G$-lattice $V_{0}$, that is, an $R$-free, finitely generated $R G$-submodule of $V$ which generates $V$ as a $K$-space. Necessarily, $\operatorname{rank}_{R} V_{0}=\operatorname{dim}_{K} V$ holds for any such $V_{0}$. Suppose now $V$ is an irreducible $K G$-module affording the "realvalued" character $\chi$ (that is, $\chi(g)=\chi\left(g^{-1}\right)$ for $\left.g \in G\right)$. Then $V$ supports a nondegenerate symmetric or alternating $G$-invariant form $($,$) and it is$ natural to ask whether some reduction $\bmod p$ has this property. Specifically, this asks for the existence of an $R G$-lattice $V_{0} \subseteq V$ with $R$-basis $\left\{v_{i}\right\}$ so that the Gram matrix $\left(\left(v_{i}, v_{j}\right)\right)$ has entries in $R$ and has determinant a unit of $R$. This last condition of course implies that $($,$) induces a nondegenerate$ $G$-invariant form on the $F G$-module $\bar{V}_{0}=V_{0} / P V_{0}$.

Let $\operatorname{OChar}(G)$ and $\operatorname{OBr}(G)$ denote the set of ordinary characters and Brauer characters afforded by $G$-modules supporting a nondegenerate symmetric bilinear form. Similarly, define $\operatorname{SkChar}(G)$ and $\operatorname{SkBr}(G)$ for the case of alternating forms. Let $\chi^{\cdot}$ denote the restriction of $\chi \in \operatorname{Char}(G)$ to $p$-regular elements. A consequence of an affirmative answer to the question of the first paragraph is that the function $\chi \mapsto \chi^{\cdot}$ sends $\operatorname{OChar}(G)$ to $\operatorname{OBr}(G)$ and $\operatorname{SkChar}(G)$ to $\operatorname{SkBr}(G)$. However, these weaker assertions are already proven in [3] when $R$ is a discrete valuation domain. (The argument is also outlined in [4].)

In fact, the proof in the DVR case amounts to finding an $R G$-lattice $V_{0}$ in $V$ so that $($,$) restricted to V_{0}$ takes on values in $R$ and induces a form on $\bar{V}_{0}$ whose radical $\bar{V}_{0}^{\perp}$ relative to this induced form is an $F G$-submodule which possesses a nondegenerate $G$-invariant form of the same type as $($,$) .$

Received by the editors May 27, 1994; a preliminary version of this paper was presented at the 22nd OSU-Denison Mathematics Conference in March 1994.

1991 Mathematics Subject Classification. Primary $20 \mathrm{C} 15$.

$K e y$ words and phrases. $G$-invariant forms, $R G$-lattice, reduction $\bmod p$. 
The question of the first paragraph essentially asks whether $V_{0}$ can always be chosen as above so that $\bar{V}_{0}^{\perp}=0$. The answer depends on the choice of $p$-modular system, which the main result of this paper shows.

Theorem. Let $K \supseteq R \rightarrow F$ be a p-modular system for $G$ with $K$ quadratically closed. If $V$ is any $K G$-module and $($,$) is a nondegenerate symmetric or$ alternating $G$-invariant form on $V$, then an $R G$-lattice $V_{0} \subseteq V$ can always be found satisfying $\left(V_{0}, V_{0}\right) \subseteq R$ and $\bar{V}_{0}^{\perp}=0$.

The result above is not always true (nor even desirable) for other choices of $p$-modular systems. For example, in establishing the basic structure of a $p$-block of defect 1 (in particular, in establishing the uniserial nature of $R G$ lattices contained in simple $K G$-modules) it appears necessary to work with representations over " $p$-minimal" fields (as defined in Goldschmidt's book [2]). In that setting, suppose $\bar{V}_{0}$ is the reduction $\bmod p$ of an $R G$-lattice in a simple module in a block of defect 1 , and assume $\bar{V}_{0}$ supports a nondegenerate $G$-invariant form. Suppose also $\bar{V}_{0}$ has two real-valued irreducible Brauer constituents. If $M \leq \bar{V}_{0}$ is a maximal locally isotropic submodule, then $M^{\perp} / M$ is semisimple of composition length 2 , violating the uniseriality of $\bar{V}_{0}$. (Explicit examples are easy to construct. Let $G=S_{3}$ be the smallest nonabelian group and $p=3$, and let $V$ be the $Q G$-module corresponding to the faithful irreducible character. In this case, $R \subseteq G$ is the ring of 3-local integers. Then $\bar{V}_{0}$ can never support a nondegenerate $G$-invariant form for any choice of $V_{0} \subseteq V$.)

On the other hand, there are settings in which it is desirable to find $V_{0} \subseteq V$ satisfying $\left(V_{0}, V_{0}\right) \subseteq R$ and $\bar{V}_{0}^{\perp}=0$. For example, if $p$ is odd and if $\mathrm{O}(W)$ denotes the full orthogonal group of a vector space over $K$ or $F$ relative to some nondegenerate symmetric form, then $\mathrm{O}(W)$ has a double cover. Hence, if $\rho: G \rightarrow \mathrm{O}(W)$ is any orthogonal representation of $G$, then the pull-back along $\rho$ determines a double cover of $G$. The cohomology class of this cover depends only on the character afforded by $W$, and we have functions $h$ : OChar $(G) \rightarrow$ $H^{2}(G,\{ \pm 1\})$ and $h: \operatorname{OBr}(G) \rightarrow H^{2}(G,\{ \pm 1\})$. If now $\chi \in \operatorname{OChar}(G)$ is afforded by $V$ and if $V_{0} \subseteq V$ can be chosen so that $\left(V_{0}, V_{0}\right) \subseteq R$ with $\bar{V}_{0}^{\perp}=0$, then the equality $h(\chi)=h\left(\chi^{\circ}\right)$ becomes transparent. This result is Theorem 6.2 of [1], except now (with the right hypothesis on the $p$-modular system) the existence of the special lattice $V_{0} \subseteq V$ is already guaranteed and need not appear as a hypothesis.

Following [4], it is convenient to introduce the following notation. Let

$$
\mathscr{L}(V)=\left\{V_{0} \mid V_{0} \text { is an } R \text {-sublattice of } V\right\}
$$

where $V$ is a finite-dimensional $K$-space. If $($,$) is a nondegenerate form on$ $V$ the collection of integral lattices is defined as

$$
\mathscr{L}_{\text {int }}(V)=\left\{V_{0} \in \mathscr{L}(V) \mid\left(V_{0}, V_{0}\right) \subseteq R\right\},
$$

while if $V$ is also a $K G$-module, set

$$
\mathscr{L}_{G}(V)=\left\{V_{0} \in \mathscr{L}(V) \mid V_{0} \text { is } G \text {-invariant }\right\} .
$$

Finally, set

$$
\mathscr{L}_{G, \text { int }}(V)=\mathscr{L}_{\text {int }}(V) \cap \mathscr{L}_{G}(V) .
$$


In the presence of a nondegenerate form, the dual $L^{*}$ of a lattice $L$ in $\mathscr{L}(V)$ is defined by $L^{*}=\{x \in V \mid(x, L) \subseteq R\}$ and, by considering dual bases, $L^{*} \in$ $\mathscr{L}(V)$. Evidently $L \subseteq L^{*}$ if and only if $L \in \mathscr{L}_{\text {int }}(V)$.

For the purposes of this note, call a lattice $L \in \mathscr{L}_{\text {int }}(V)$ " $c$-homogeneous" if the Gram matrix of this form computed with respect to some (hence any) $R$-basis of $L$ has the form $c \cdot U$ for some nonsingular matrix $U$ over $R$ whose inverse also has entries in $R$. Notice that by consideration of dual bases we have $L^{*}=\frac{1}{c} L$ for $c$-homogeneous lattices (and $L^{*}$ is $\frac{1}{c}$-homogeneous).

It is convenient to start with an elementary lemma (which is essentially the Gram-Schmidt process).

Lemma. Let (, ) be a nondegenerate form defined on the $K$-space $V$, and let $L$ be any integral $R$-sublattice of $V$. Then there exists an orthogonal decomposition of $L$ as

$$
L=W_{1} \perp W_{2} \perp \cdots \perp W_{k}
$$

where each $W_{i}$ is $c_{i}$-homogeneous for some $c_{i} \neq 0$ and $R \supseteq\left(c_{1}\right) \supseteq\left(c_{2}\right) \supseteq \cdots \supseteq$ $\left(c_{k}\right)$.

Proof. The set $(L, L) \subseteq R$ is an ideal of $R$ which is finitely generated (by the entries of the Gram matrix with respect to some basis) and so it is principally generated, say by $c_{1} \in R$. Let $(,)^{\prime}$ denote the form $\frac{1}{c_{1}}($,$) . Then$ ( , )' induces a nonzero form on $\bar{L}=L / P L$, whose radical $\bar{L}^{\perp}$ therefore is proper in $\bar{L}$. Write $d=\operatorname{dim}_{F} \bar{L} / \bar{L}^{\perp}$ and choose $v_{1}, v_{2}, \ldots, v_{d} \in L$ so that the natural image of $\left\{v_{1}, v_{2}, \ldots, v_{d}\right\}$ in $\bar{L} / L^{\perp}$ is an $F$-basis. If $W_{1}=\sum_{i=1}^{d} R v_{i}$, then the original form restricted to $W_{1}$ is nondegenerate, and $W_{1}$ is $c_{1}$-homogeneous by construction. Thus, if $\left\{v_{1}^{*}, v_{2}^{*}, \ldots, v_{d}^{*}\right\}$ denotes the dual basis of $\left\{v_{1}, v_{2}, \ldots, v_{d}\right\}$ in $K W_{1}$, then $v_{i}^{*} \in \frac{1}{c_{1}} W_{1}$ for every $i$. Hence, if $x \in L$, then $\left(x, v_{i}\right) v_{i}^{*} \in W_{1}$ for each $i$, so that $x^{\prime}=x-\sum\left(x, v_{i}\right) v_{i}^{*}$ belongs to $L$ and $\left(x^{\prime}, W_{1}\right)=0$. This shows $L=W_{1} \perp Y$ where $Y=\{x \in$ $\left.L \mid\left(x, W_{1}\right)=0\right\}$.

This construction may be continued in the $R$-submodule $Y$, producing the decomposition of the lemma, as required.

Notice that if $L=W_{1} \perp W_{2} \perp \cdots \perp W_{k}$ as in the lemma, then

$$
L^{*}=\frac{1}{c_{1}} W_{1} \perp \frac{1}{c_{2}} W_{2} \perp \cdots \perp \frac{1}{c_{k}} W_{k} .
$$

It is possible to refine the decomposition so that $\operatorname{rank}_{R}\left(W_{i}\right) \leq 2$ for all $i$ and even $\operatorname{rank}_{R}\left(W_{i}\right)=1$ in the symmetric case when $p$ is odd.

Proof of Theorem. If $L \in \mathscr{L}_{G \text {, int }}(V)$ define $r(L)$ to be the rank over $F$ of the matrix obtained from the Gram matrix of $($,$) by reducing the entries mod P$. Select $L \in \mathscr{L}_{G \text {,int }}(V)$ so that $r(L)$ is maximal, and let $L=W_{1} \perp W_{2} \perp \cdots \perp W_{k}$ be the decomposition of $L$ as in the previous lemma. We may clearly assume, using the notation of that lemma, that each inclusion $\left(c_{i+1}\right) \subset\left(c_{i}\right)$ is strict. If $\left(c_{1}\right) \neq R$, then $r(L)=0, \frac{1}{\sqrt{c_{1}}} L \in \mathscr{L}_{G \text {, int }}(V)$ and $r\left(\frac{1}{\sqrt{c_{1}}} L\right)>0$, for a contradiction. If $\left(c_{k}\right) \neq R$ let $V_{1}=L+\sqrt{c_{k}} L^{*} \in \mathscr{L}_{G}(V)$. Then $V_{1}=X_{1} \perp$ $X_{2} \perp \cdots \perp X_{k}$, where

$$
X_{i}=W_{i}+\frac{\sqrt{c_{k}}}{c_{i}} W_{i}= \begin{cases}W_{i} & \text { if } \sqrt{c_{k}} \in\left(c_{i}\right), \\ \frac{\sqrt{c_{k}}}{c_{i}} W_{i} & \text { if } \sqrt{c_{k}} \notin\left(c_{i}\right) .\end{cases}
$$


Clearly,

$$
\left(X_{i}, X_{i}\right)=\left(W_{i}, W_{i}\right)=\left(c_{i}\right) \subseteq R \text { if } \sqrt{c_{k}} \in\left(c_{i}\right)
$$

while

$$
\left(X_{i}, X_{i}\right)=\left(\frac{\sqrt{c_{k}}}{c_{i}} W_{i}, \frac{\sqrt{c_{k}}}{c_{i}} W_{i}\right)=\frac{c_{k}}{c_{i}^{2}}\left(c_{i}\right) \subseteq R \text { if } \sqrt{c_{k}} \notin\left(c_{i}\right) .
$$

This proves that $V_{1} \in \mathscr{L}_{G \text {,int }}(V)$, and at the same time $\left(X_{i}, X_{i}\right)=R$ for both $i=1$ and $k$. As each $X_{i}$ is homogeneous we conclude that $r\left(V_{1}\right) \geq$ $\operatorname{rank}_{R}\left(X_{1}\right)+\operatorname{rank}_{R}\left(X_{k}\right)>\operatorname{rank}_{R}\left(X_{1}\right)=r(L)$, for another contradiction.

Thus $\left(c_{i}\right)=R$ for all $i$, proving the theorem.

\section{REFERENCES}

1. S. M. Gagola, Jr. and S. C. Garrison, III, Real characters, double covers, and the multiplier. II, J. Algebra 98 (1986), 38-75.

2. D. M. Goldschmidt, Lectures on character theory, Publish or Perish, Berkeley, CA, 1980.

3. D. Quillen, The Adams conjecture, Topology 10 (1971), 67-80.

4. J. G. Thompson, Bilinear forms in characteristic $p$ and the Frobenius-Schur indicator, Group Theory (Beijing 1984), Lecture Notes in Math., vol. 1185, Springer-Verlag, Berlin and New York, 1985, pp. 221-230.

Department of Mathematics and Computer Science, Kent State University, Kent, ОнІо 44242

E-mail address: gagola@mcs. kent. edu 